\title{
Skin and soft tissue infections in hospitalised patients with diabetes: culture isolates and risk factors associated with mortality, length of stay and cost
}

\author{
B. A. Lipsky • Y. P. Tabak • R. S. Johannes $・$ L. Vo • \\ L. Hyde • J. A. Weigelt
}

Received: 17 September 2009 / Accepted: 4 January 2010/Published online: 10 February 2010

(C) US Government 2010

\begin{abstract}
Aims/hypothesis Skin and soft tissue infections (SSTIs) cause substantial morbidity in persons with diabetes. There are few data on pathogens or risk factors associated with important outcomes in diabetic patients hospitalised with SSTIs.

Methods Using a clinical research database from CareFusion, we identified 3,030 hospitalised diabetic patients with positive culture isolates and a diagnosis of SSTI in 97 US hospitals between 2003 and 2007. We classified the culture isolates and analysed their association with the anatomic location of infection, mortality, length of stay and hospital costs.

Results The only culture isolate with a significantly increased prevalence was methicillin-resistant Staphylococcus aureus (MRSA); prevalence for infection of the foot was increased from 11.6 to $21.9 \%(p<0.0001)$ and for non-foot
\end{abstract}

B. A. Lipsky $(\bowtie)$

VA Puget Sound Health Care System,

General Internal Medicine (S-111-PCC), University of Washington,

1660 S. Columbian Way,

Seattle, WA, USA

e-mail: balipsky@uw.edu

Y. P. Tabak $\cdot$ R. S. Johannes $\cdot$ L. Hyde

Clinical Research, CareFusion,

Marlborough, MA, USA

R. S. Johannes

Division of Gastroenterology, Harvard Medical School,

Boston, MA, USA

L. Vo

Ortho-McNeil Janssen Scientific Affairs, LLC,

Raritan, NJ, USA

J. A. Weigelt

Department of Surgery, Medical College of Wisconsin,

Milwaukee, WI, USA locations from $14.0 \%$ to $24.6 \%(p=0.006)$. Patients with non-foot (vs foot) infections were more severely ill at presentation and had higher mortality rates $(2.2 \%$ vs $1.0 \%$, $p<0.05)$. Significant independent risk factors associated with higher mortality rates included having a polymicrobial culture with Pseudomonas aeruginosa (OR 3.1), a monomicrobial culture with other gram-negatives (OR 8.9), greater illness severity (OR 1.9) and being transferred from another hospital (OR 5.1). These factors and need for major surgery were also independently associated with longer length of stay and higher costs.

Conclusions/interpretation Among diabetic patients hospitalised with SSTI from 2003 to 2007, only MRSA increased in prevalence. Patients with non-foot (vs foot) infections were more severely ill. Independent risk factors for increased mortality rates, length of stay and costs included more severe illness, transfer from another hospital and wound cultures with Pseudomonas or other gram-negatives.

Keywords Diabetes $\cdot$ Foot infections $\cdot$ Hospitalised patients $\cdot$ Length of stay · Mortality · MRSA ·

Skin and soft tissue infection

\section{Abbreviations \\ MRSA Methicillin-resistant Staphylococcus aureus \\ MSSA Methicillin-susceptible Staphylococcus aureus \\ SSTI Skin and soft tissue infection}

\section{Introduction}

Skin and soft tissue infections (SSTIs) are leading causes of morbidity and occasionally mortality in persons with 
diabetes mellitus [1-4]. Various complications of diabetes (e.g. sensory neuropathy, vascular insufficiency, immunopathy and metabolic perturbations) can predispose these patients to infections $[5,6]$. SSTIs are more common and more severe in diabetic than in non-diabetic patients and constitute a leading cause of hospitalisation [7]. The risk of SSTI-related hospitalisation is more than twice as high in diabetic than in non-diabetic patients [8]. Diabetes is independently associated with increased emergency department visits for SSTIs [9], longer hospital stays [8] and infection-attributable death $[10,11]$. The rising prevalence of diabetes $[7,12]$ is likely to cause increasing numbers of diabetes-related SSTIs in hospitalised patients.

SSTIs occur at all anatomic sites, but the foot is most frequently affected in diabetic patients [7]. In the USA, about 111,000 persons with diabetes are hospitalised annually with foot infections and these precede and contribute to almost $60 \%$ of all lower extremity amputations [13]. Staphylococcus aureus is the most commonly isolated pathogen in SSTIs, both in ambulatory and hospitalised patients [14-16]. Antimicrobial-resistant pathogens, especially methicillin-resistant $S$. aureus (MRSA), are increasingly isolated in nosocomial infections [17-20]. Several recent reports identified MRSA as the leading pathogen in SSTIs [21-24]; it also causes $20 \%$ to $50 \%$ of diabetes-associated foot infections in several countries and is associated with worse outcomes than other pathogens [23-29].

The few published multi-centre studies characterising SSTI pathogens have been limited to specific types of organisms [30], with almost all reports on SSTIs in diabetic patients limited to foot infections [31-33]. Few studies have addressed the interaction of specific pathogens with various clinical and economic outcomes [34]. To understand the current characteristics of these common and potentially severe infections better, we investigated the epidemiology and microbiology of wounds diagnosed as SSTI of all anatomic sites in hospitalised diabetic patients. We also assessed the association of infection at various anatomic locations with the organisms isolated from wound cultures, clinical outcomes and hospital costs.

\section{Methods}

Data source Data for this study were from one of the clinical research databases of CareFusion (CareFusion Clinical Research Services, Marlborough, MA, USA), which has been used for research for over two decades and been fully described elsewhere [35-39]. Briefly, the database captures selected clinical (e.g. vital signs), laboratory (chemistry, haematology and culture) and administrative (patient demographics, diagnoses, duration of hospitalisation, discharge status, total charges) data. The data for this study were derived from a dataset with all patient-specific information anonymised. It was conducted in compliance with US federal regulations, the Health Insurance Portability and Accountability Act (HIPAA) and the Helsinki Declaration.

Study population We reviewed data on patients admitted to 97 selected hospitals primarily located in the Northeast region of the United States between January 2003 and June 2007 to identify those with a principal diagnosis (International Classification of Diseases, Ninth Revision, Clinical Modification [ICD-9-CM]) of diabetes and a secondary diagnosis indicating any type of SSTI (including cellulitis, infected ulcer or surgical site infection) involving the foot or any other anatomic site. Included patients had to have a positive result from a culture of an infected skin or soft tissue site (many of which were open wounds) taken within $48 \mathrm{~h}$ of hospitalisation.

Definition and classification of type of infection Based on their secondary diagnoses and the culture specimen site, we classified each eligible patient as having: cellulitis, an infected skin ulcer or a surgical site infection. We further identified infections as affecting the foot or another (nonfoot) site, classifying infections that involved both sites in the foot group.

Definition and classification of isolated organism(s) Based on wound culture results, we classified patients by the number of isolates (monomicrobial or polymicrobial) and by the specific organism(s) isolated. Because of the implications for selecting appropriate antibiotic therapy for infections caused by MRSA and Pseudomonas aeruginosa, we hierarchically classified polymicrobial cultures into one of three mutually exclusive categories: (1) MRSA polymicrobial (MRSA + any other organism); (2) Pseudomonas polymicrobial ( $P$. aeruginosa + any non-MRSA organism); or (3) other polymicrobial (caused by two or more organisms other than MRSA or P. aeruginosa).

Outcome measures For each patient we recorded any inhospital mortality, length of stay in the hospital and the total costs for the index hospitalisation. We estimated the hospitalisation costs from billed total charges and calculated the hospital-specific cost/charge ratio for each pertinent calendar year using data obtained from the Centers for Medicare and Medicaid Services [40].

Statistical analysis After categorising the micro-organisms isolated from each wound, we analysed the incidence of all isolates over the study period, as well as the types of pathogens found in infections of the foot compared with 
non-foot sites. We determined the crude mortality rate, length of stay and hospitalisation costs for infections associated with each organism. We performed statistical analyses in SAS (version 9.01; SAS Institute, Cary, NC, USA) using Fisher's exact test for dichotomous variables due to low mortality events, Wilcoxon non-parametric test for continuous variables and the Cochran-Armitage statistic for trending analysis.

We categorised admission illness severity using an aggregated score, which was generated from the CareFusion multivariable mortality predictive models and adjusts for demographics, physiological presentation and co-morbidities [39]. We also captured other variables that might have had potentially confounding effects on clinical presentation or economic burden, including source of admission (e.g. transfer from another acute care hospital or a skilled nursing facility), any hospitalisation within the previous 30 days or need for a major surgical procedure (i.e. involving spinal or general anaesthesia) at the index hospitalisation. We then conducted forward stepwise logistic regression analyses for mortality and general linear models for log-transformed length of stay and cost to determine the independent contributions of each isolated organism. For the length of stay and cost outcomes, the distributions of which tended to be skewed, we used a $\log$ scale. We re-transformed the adjusted $\log$ scale length of stay and cost to the natural units of days and US dollars, using the Smearing estimate for correction of bias [41]. We used the median from 1,000 bootstrap reiterations as the retransformed estimate for the attributable length of stay and cost with the 2.5th and 97.5th percentiles for the $95 \% \mathrm{CI}$ estimates [42].

\section{Results}

Baseline patient characteristics A total of 3,030 patients met our inclusion criteria. Since the number with surgical site infections was relatively small $(n=114)$ and the anatomic locations were not available, we lumped these patients into the non-foot infection group, with whom their clinical severity and outcomes were compatible, for aggregated descriptive analyses. The median age (and interquartile range) was similar for patients with a foot infection and a non-foot infection ( $p=0.50$; Table 1). Compared with those with a foot infection, patients in the non-foot infection group were significantly less likely to be male $(54.6 \%$ vs $64.0 \%, p<0.0001)$ and significantly more likely to have had a previous admission at the same hospital within the prior 30 days $(11.2 \%$ vs $8.7 \%, p<0.05)$ or to have come from a skilled nursing facility $(5.3 \%$ vs $2.8 \%$, $p<0.05$ ). They were also more likely to have had a physiological derangement on admission, including hypoglycaemia $(7.0 \%$ vs $3.5 \%, p<0.001)$ and brady- or tachycardia $(10.5 \%$ vs $3.0 \%, p<0.0001)$, as well as altered mental status $(12.5 \%$ vs $6.3 \%, p<0.0001)$, hypoalbuminaemia $(30.3 \%$ vs $22.0 \%, p<0.001)$ and elevated bands on the leucocyte differential $(22.3 \%$ vs $13.1 \%, p<0.01)$. A total of $84 \%$ of patients had at least one comorbidity. Those that differed significantly for non-foot compared with foot infection patients were those with: congestive heart failure (27.0\% vs $21.4 \%, p<0.01)$; chronic lung disease $(13.2 \%$ vs $10.0 \%, p<0.05)$; chronic renal failure $(23.1 \%$ vs $18.8 \%, p<$ $0.05)$; and a history of amputation $(22.0 \%$ vs $29 \%, p<0.001)$. The highest quartile of aggregated clinical severity contained more patients with non-foot infection than with foot infection $(29.5 \%$ vs $23.3 \%, p<0.01)$.

Pathogens isolated Wound cultures revealed polymicrobial isolates in $53.0 \%$ of non-foot and in $58.7 \%$ of foot lesions (Table 2). Compared with lesions diagnosed as foot infections, non-foot infections were more likely to have MRSA $(9.6 \%$ vs $7.4 \%, p=0.06)$ or methicillin-susceptible S. aureus (MSSA; $16.4 \%$ vs $13.2 \%, p<0.05$ ), but less likely to have isolates of Streptococcus spp $(4.3 \%$ vs $7.2 \%, p<$ 0.01 ) or polymicrobial isolates without MRSA or $P$. aeruginosa $(33.7 \%$ vs $41.8 \%, p<0.001)$.

Over the study period, the proportion of isolates of MRSA increased for non-foot (from $14.0 \%$ to $24.6 \%, p=$ 0.006 for trend) and foot (from $11.6 \%$ to $21.9 \%, p<0.0001$ for trend) sites (Fig. 1). Both monomicrobial and polymicrobial MRSA wound isolates increased significantly, while those with MSSA did not. In addition, the proportion of polymicrobial isolates not involving MRSA or $P$. aeruginosa significantly declined (from $46.4 \%$ to $31.7 \%$, $p<0.0001$ for trend). There were no significant changes in proportion of isolates of any other pathogens.

Mortality, length of stay and cost outcomes in univariate analyses Overall, $41(1.4 \%)$ of the patients in the study population died during hospitalisation (Table 3). Factors significantly associated with increased mortality rate were: severity of illness on admission $(p<0.0001)$; type of infection (cellulitis vs ulcer vs surgical site, $p<0.001$ ); and non-foot location $(p<0.05)$. Wounds with polymicrobial isolates that included $P$. aeruginosa and monomicrobial cultures with other (i.e. non-Pseudomonas) gram-negatives were each associated with significantly increased mortality rates $(p<0.01$ and $p<0.001$, respectively). Factors significantly associated with greater length of stay and higher costs were: increased severity of illness; having been transferred from another acute care hospital or skilled nursing facility; or culture results showing monomicrobial other gram-negative or $P$. aeruginosa, or polymicrobial MRSA.

Independent risk factors for mortality, length of stay and cost in multivariable analyses In multivariable logistic 
Table 1 Baseline characteristics of diabetic patients hospitalised for skin and skin structure infections

\begin{tabular}{|c|c|c|c|c|c|c|c|}
\hline \multirow[t]{3}{*}{ Variable } & \multicolumn{7}{|c|}{ Type of infection } \\
\hline & \multicolumn{2}{|l|}{ Cellulitis } & \multicolumn{3}{|l|}{ Ulcer/other } & \multicolumn{2}{|l|}{ Overall } \\
\hline & Foot & Non-foot & Foot & Non-foot & $\mathrm{SSI}^{\mathrm{a}}$ & Foot & $\begin{array}{l}\text { Non-foot or } \\
\text { SSI }^{\mathrm{a}}\end{array}$ \\
\hline$n$ & 1,828 & 597 & 392 & 99 & 114 & 2,220 & 810 \\
\hline \multicolumn{8}{|l|}{ Demographics } \\
\hline Age (years) ${ }^{\mathrm{b}}$ & $60(50-70)$ & $59(48-71)$ & $59(50-72)$ & $64(47-78)$ & $63(54-74)$ & $60(50-70)$ & $61(49-72)$ \\
\hline Death during hospitalisation & $16(0.9)$ & $7(1.2)$ & $7(1.8)$ & $4(4.0)$ & $7(6.1)$ & $23(1.0)$ & $18(2.2) *$ \\
\hline Male sex & $1,166(63.8)$ & $328(54.9)^{* * *}$ & $254(64.8)$ & $47(47.5)^{* *}$ & $67(58.8)$ & $1,420(64.0)$ & $442(54.6)^{* * * *}$ \\
\hline \multicolumn{8}{|l|}{ Hospitalisation and transfer status } \\
\hline Prior admission to same hospital ${ }^{\mathrm{c}}$ & $143(7.8)$ & $47(7.9)$ & $50(12.8)$ & $22(22.2)^{*}$ & $22(19.3)$ & $193(8.7)$ & 91 (11.2)* \\
\hline $\begin{array}{l}\text { Transfer from other acute-care } \\
\text { hospital }\end{array}$ & $13(0.7)$ & $3(0.5)$ & $8(2.0)$ & $1(1.0)$ & $2(1.8)$ & $21(0.9)$ & $6(0.7)$ \\
\hline $\begin{array}{l}\text { Transfer from skilled nursing } \\
\text { facility }\end{array}$ & $42(2.3)$ & $19(3.2)$ & $20(5.1)$ & $13(13.1)$ & $11(9.6)$ & $62(2.8)$ & $43(5.3)^{* *}$ \\
\hline \multicolumn{8}{|l|}{ Acute clinical presentation ${ }^{\mathrm{d}}$} \\
\hline Systolic BP $<100 \mathrm{mmHg}$ & $191(10.4)$ & $99(16.6)^{* * *}$ & $72(18.4)$ & $26(26.3)$ & $18(15.8)$ & $263(11.8)$ & $143(17.7)^{* * * *}$ \\
\hline Temperature $<35.6$ or $\geq 38.0^{\circ} \mathrm{C}$ & $474(25.9)$ & $154(25.8)$ & $202(51.5)$ & $49(49.5)$ & $46(40.4)$ & $676(30.5)$ & $249(30.7)$ \\
\hline Pulse $<49$ or $>125$ beats $/ \mathrm{min}$ & $57(3.1)$ & $61(10.2)^{* * * *}$ & $26(6.6)$ & $14(14.1)^{*}$ & $10(8.8)$ & $83(3.7)$ & $85(10.5)^{* * * *}$ \\
\hline $\begin{array}{l}\text { Respiration }<10 \text { or }>29 \text { breaths/ } \\
\text { min }\end{array}$ & $44(2.4)$ & $43(7.2)^{* * * *}$ & $23(5.9)$ & $5(5.1)$ & $7(6.1)$ & $67(3.0)$ & $55(6.8)^{* * * *}$ \\
\hline Altered mental status & $85(4.6)$ & $50(8.4)^{* * *}$ & $54(13.8)$ & $29(29.3)^{* * *}$ & $22(19.3)$ & $139(6.3)$ & $101(12.5)^{* * * *}$ \\
\hline \multicolumn{8}{|l|}{ Laboratory results } \\
\hline Albumin $\leq 27 \mathrm{~g} / 1$ & $151(19.4)$ & $81(26.1)^{*}$ & $58(33.5)$ & $25(44.6)$ & $19(41.3)$ & $209(22)$ & $125(30.3)^{* *}$ \\
\hline $\begin{array}{l}\text { Blood urea nitrogen } \\
>14.3 \mathrm{mmol} / 1\end{array}$ & $285(16.5)$ & 98 (17.6) & $82(22.2)$ & $32(33.7)^{*}$ & $25(23.2)$ & $367(17.5)$ & $155(20.4)$ \\
\hline Creatinine $>228.8 \mu \mathrm{mol} / 1$ & $118(6.9)$ & $41(7.4)$ & $53(14.4)$ & $10(10.5)$ & $22(20.4)$ & $171(8.2)$ & $73(9.6)$ \\
\hline Sodium $\geq 146 \mathrm{mmol} / 1$ & $10(0.6)$ & $4(0.7)$ & $8(2.2)$ & $10(10.5)^{* * *}$ & $3(2.8)$ & $18(0.9)$ & $17(2.2)^{* *}$ \\
\hline Total bilirubin $>13.7 \mu \mathrm{mol} / 1$ & $152(20.2)$ & $72(23.8)$ & $45(27.4)$ & $13(23.2)$ & $14(34.2)$ & $197(21.5)$ & $99(24.8)$ \\
\hline $\mathrm{O}_{2}$ pressure /saturation ${ }^{\mathrm{e}}$ & $6(19.4)$ & $17(32.7)$ & $6(26.1)$ & $8(53.3)$ & $4(26.7)$ & $12(22.2)$ & $29(35.4)$ \\
\hline PT INR $>1.2$ or PT $>14 \mathrm{~s}$ & $140(17.7)$ & $43(16.9)$ & $55(27.8)$ & $13(27.1)$ & $19(29.7)$ & $195(19.7)$ & $75(20.5)$ \\
\hline $\begin{array}{l}\text { Leucocyte differential }>0.13 \\
\text { bands }\end{array}$ & $51(14.5)$ & $31(21.8)$ & $13(9.4)$ & $9(28.1)^{* *}$ & $6(18.8)$ & $64(13.1)$ & $46(22.3)^{* *}$ \\
\hline White cell count $\geq 11 \times 10^{9} / 1$ & $790(45.6)$ & $247(43.6)$ & $272(72.9)$ & $60(62.5)$ & $70(66.7)$ & $1,062(50.5)$ & $377(49.2)$ \\
\hline Physiological derangements $\geq 1^{\mathrm{f}}$ & $1,082(59.2)$ & $360(60.3)$ & $324(82.7)$ & $83(83.8)$ & $86(75.4)$ & $1,406(63.3)$ & $529(65.3)$ \\
\hline \multicolumn{8}{|l|}{ Glucose on admission $(\mathrm{mmol} / \mathrm{l})$} \\
\hline$\leq 3.9$ & $54(3.0)$ & $37(6.2)^{* *}$ & $24(6.1)$ & $8(8.1)$ & $12(10.5)$ & $78(3.5)$ & $57(7.0)^{* * *}$ \\
\hline $3.94-7.5$ & $288(15.8)$ & $93(15.6)$ & $51(13.0)$ & $9(9.1)$ & $18(15.8)$ & $339(15.3)$ & $120(14.8)$ \\
\hline $7.55-13.3$ & $596(32.6)$ & $169(28.3)$ & $124(31.7)$ & $26(26.3)$ & $41(36)$ & $720(32.4)$ & $236(29.1)$ \\
\hline$>13.3$ & $890(48.7)$ & $298(49.9)$ & $193(49.2)$ & $56(56.6)$ & $43(37.7)$ & $1,083(48.8)$ & $397(49.0)$ \\
\hline \multicolumn{8}{|l|}{ Co-morbidities } \\
\hline Congestive heart failure & $381(20.8)$ & $152(25.5)^{*}$ & $95(24.2)$ & $34(34.3)$ & $33(28.9)$ & $476(21.4)$ & $219(27.0)^{* *}$ \\
\hline History MCI & $155(8.5)$ & $42(7.0)$ & $39(9.9)$ & $5(5.1)$ & $16(14.0)$ & $194(8.7)$ & $63(7.8)$ \\
\hline Prior coronary artery intervention & $350(19.1)$ & $110(18.4)$ & $90(23.0)$ & $10(10.1)^{* *}$ & $32(28.1)$ & $440(19.8)$ & $152(18.8)$ \\
\hline Immunosuppressive medication & $51(2.8)$ & $22(3.7)$ & $22(5.6)$ & $7(7.1)$ & $5(4.4)$ & $73(3.3)$ & $34(4.2)$ \\
\hline Cancer & $33(1.8)$ & $8(1.3)$ & $12(3.1)$ & $3(3.0)$ & $2(1.8)$ & $45(2.0)$ & $13(1.6)$ \\
\hline Peripheral vascular disease & $633(34.6)$ & $181(30.3)$ & $189(48.2)$ & $38(38.4)$ & $62(54.4)$ & $822(37.0)$ & $281(34.7)$ \\
\hline Chronic liver disease & $21(1.1)$ & $8(1.3)$ & $9(2.3)$ & $2(2.0)$ & $0(0.0)$ & $30(1.4)$ & $10(1.2)$ \\
\hline Chronic lung disease & $170(9.3)$ & $81(13.6)^{* *}$ & $53(13.5)$ & $11(11.1)$ & $15(13.2)$ & $223(10.0)$ & $107(13.2)^{*}$ \\
\hline Previous stroke & $170(9.3)$ & $62(10.4)$ & $48(12.2)$ & $14(14.1)$ & $16(14.0)$ & $218(9.8)$ & $92(11.4)$ \\
\hline Chronic renal disease & $325(17.8)$ & $120(20.1)$ & $93(23.7)$ & $25(25.3)$ & $42(36.8)$ & $418(18.8)$ & 187 (23.1)* \\
\hline
\end{tabular}


Table 1 (continued)

\begin{tabular}{|c|c|c|c|c|c|c|c|}
\hline \multirow[t]{3}{*}{ Variable } & \multicolumn{7}{|c|}{ Type of infection } \\
\hline & \multicolumn{2}{|l|}{ Cellulitis } & \multicolumn{3}{|l|}{ Ulcer/other } & \multicolumn{2}{|l|}{ Overall } \\
\hline & Foot & Non-foot & Foot & Non-foot & $\mathrm{SSI}^{\mathrm{a}}$ & Foot & $\begin{array}{l}\text { Non-foot or } \\
\text { SSI }^{\mathrm{a}}\end{array}$ \\
\hline History of amputation & $526(28.8)$ & $98(16.4)^{* * * *}$ & $120(30.6)$ & $22(22.2)$ & $58(50.9)$ & $646(29.1)$ & $178(22.0)^{* * * *}$ \\
\hline At least one comorbidity & $1,510(82.6)$ & $485(81.2)$ & $349(89.0)$ & $85(85.9)$ & $109(95.6)$ & $1,859(83.7)$ & $679(83.8)$ \\
\hline \multicolumn{8}{|l|}{ Admission illness severity ${ }^{\mathrm{g}}$} \\
\hline 1st quartile & $513(28.1)$ & $163(27.3)$ & $55(14.0)$ & $12(12.1)$ & $15(13.2)$ & $568(25.6)$ & $190(23.5)^{* *}$ \\
\hline 2nd quartile & $506(27.7)$ & $143(24.0)$ & $77(19.6)$ & $15(15.2)$ & $17(14.9)$ & $583(26.3)$ & $175(21.6)$ \\
\hline 3rd quartile & $450(24.6)$ & $153(25.6)$ & $101(25.8)$ & $24(24.2)$ & $29(25.4)$ & $551(24.8)$ & $206(25.4)$ \\
\hline 4th quartile & $359(19.6)$ & $138(23.1)$ & $159(40.6)$ & $48(48.5)$ & $53(46.5)$ & $518(23.3)$ & $239(29.5)$ \\
\hline
\end{tabular}

Data are shown as $n(\%)$ unless otherwise specified. For laboratory data, \% means \% of those with respective laboratory variable measured ${ }^{a}$ SSI, surgical site infection at any anatomic location; ${ }^{b}$ median interquartile range (IQR); ${ }^{\mathrm{c}} 30$ or fewer days previously; ${ }^{\mathrm{d}}$ vital signs and altered mental status; ${ }^{e}$ partial $\mathrm{O}_{2}$ pressure $<55$ or $>140 \mathrm{mmHg}$ or $\mathrm{O}_{2}$ saturation $<90 \%$; ${ }^{\text {f }}$ excluding glucose

${ }^{g}$ Severity was calculated as predicted probability of death on admission based on a multivariable logistic regression model including age, physiological and comorbidity variables listed in this table. For glucose and admission severity, $p$ values represent an overall multilevel $\chi^{2}$ test. For all other dichotomous variables, $p$ value represents a pair-wise $\chi^{2}$ test

For all variables, significance tests were conducted between those with a foot vs those with a non-foot infection, ${ }^{*} p<0.05,{ }^{* *} p<0.01,{ }^{* * *} p<0.001$ and $* * * * p<0.0001$

MCI, myocardial infarction; PT, prothrombin time; PT INR, prothrombin time/international normalised ratio

analyses the significant independent predictors for inhospital mortality were: increased illness severity; transfer from another acute care hospital; surgical site infection; and cultures yielding other gram-negative or polymicrobial $P$. aeruginosa (Table 4). Factors independently associated with greater length of stay and higher costs were: polymicrobial cultures including $P$. aeruginosa or monomicrobial infection with other gram-negative; greater illness severity; transfer from another hospital; and need for major surgery. The incremental length of stay and costs for polymicrobial $P$. aeruginosa were 1.2 days (95\% CI $0.4-$ 1.9 ) and US $\$ 2,403$ (95\% CI $\$ 1,068-3,822)$; for other gramnegatives, they were 2.2 days $(95 \%$ CI $0.8-3.9)$ and $\$ 3,256$ (95\% CI \$1,076-5,770). Interestingly, after adjusting for illness severity and other confounders, the differences in mortality rates, length of stay or cost between patients who had a foot infection and those with a non-foot infection were not statistically significant.

\section{Discussion}

This analysis of data from a large group of hospitalised diabetic patients with a clinical diagnosis of SSTI and a positive culture from a soft tissue sample enabled us to make several important observations. As expected, the foot was the most frequently involved site, but over a quarter of the diagnosed infections involved other anatomic sites. This is noteworthy because most publications on soft tissue infections in persons with diabetes deal only with foot infections. While we found many similarities between patients diagnosed with an infection of the foot vs another site, there were some significant differences.

As noted in other studies [5, 13], almost two-thirds of our patients with a foot infection were men, but men comprised just over one-half of the patients with non-foot infections. However, patients with a non-foot infection were more likely to have been recently hospitalised or to be residing in a skilled nursing facility, and were more severely ill on admission with higher rates of various physiological derangements. This latter finding may be explained by the fact that, due to concern about risk of lower extremity amputation, patients may be hospitalised with lesser severity foot than non-foot infections. Alternatively, while foot infections are generally a consequence of complications caused by peripheral neuropathy, infection at a non-foot site may be more likely to occur in a patient with impaired immunity or co-morbidities. This possibility is supported by the fact that more patients with a non-foot infection had a history of congestive heart failure, chronic lung disease and chronic renal disease.

Cultures of SSTI showed that staphylococci were the most frequent causative isolates. While MSSA was the most common isolate, $8 \%$ of patients had MRSA. Only the prevalence of MRSA isolates increased significantly in patients with a positive culture during the study period, this 
Table 2 Microbial isolates from SSTI sites in hospitalised diabetic patients

\begin{tabular}{|c|c|c|c|c|c|c|c|}
\hline \multirow[t]{3}{*}{ Variable } & \multicolumn{7}{|c|}{ Type of infection } \\
\hline & \multicolumn{2}{|l|}{ Cellulitis } & \multicolumn{3}{|c|}{ Ulcer/other infection } & \multicolumn{2}{|l|}{ Overall } \\
\hline & Foot & Non-foot & Foot & Non-foot & $\mathrm{SSI}^{\mathrm{a}}$ & Foot & Non-foot or $\mathrm{SSI}^{\mathrm{a}}$ \\
\hline Patients $(n)$ & 1,828 & 597 & 392 & 99 & 114 & 2,220 & 810 \\
\hline \multicolumn{8}{|l|}{ Monomicrobial, gram-positive ${ }^{\mathrm{b}}$} \\
\hline MSSA & $259(14.2)$ & $114(19.1)^{* *}$ & $35(8.9)$ & $8(8.1)$ & $11(9.6)$ & $294(13.2)$ & $133(16.4)^{*}$ \\
\hline MRSA & $139(7.6)$ & $54(9.0)$ & $26(6.6)$ & $11(11.1)$ & $13(11.4)$ & $165(7.4)$ & $78(9.6)$ \\
\hline $\mathrm{CoN}^{\mathrm{c}}$ staphylococci & $112(6.1)$ & $40(6.7)$ & $11(2.8)$ & $5(5.1)$ & $3(2.6)$ & $123(5.5)$ & $48(5.9)$ \\
\hline Streptococcus spp. & $137(7.5)$ & $27(4.5)^{*}$ & $23(5.9)$ & $4(4.0)$ & $4(3.5)$ & $160(7.2)$ & $35(4.3)^{* *}$ \\
\hline Enterococcus spp. & $37(2.0)$ & $14(2.3)$ & $9(2.3)$ & $0(0.0)$ & $4(3.5)$ & $46(2.1)$ & $18(2.2)$ \\
\hline Other gram-positive & $6(0.3)$ & $5(0.8)$ & $1(0.3)$ & $0(0.0)$ & $0(0.0)$ & $7(0.3)$ & $5(0.6)$ \\
\hline \multicolumn{8}{|l|}{ Monomicrobial, gram-negative ${ }^{b}$} \\
\hline Enterobacter & $7(0.4)$ & $2(0.3)$ & $2(0.5)$ & $1(1.0)$ & $1(0.9)$ & $9(0.4)$ & $4(0.5)$ \\
\hline P. aeruginosa & $22(1.2)$ & $11(1.8)$ & $8(2.0)$ & $1(1.0)$ & $4(3.5)$ & $30(1.4)$ & $16(2.0)$ \\
\hline Other gram-negative & $53(2.9)$ & $21(3.5)$ & $12(3.1)$ & $4(4.0)$ & $5(4.4)$ & $65(2.9)$ & $30(3.7)$ \\
\hline Anaerobic bacteria & $10(0.5)$ & $4(0.7)$ & $1(0.3)$ & $0(0.0)$ & $1(0.9)$ & $11(0.5)$ & $5(0.6)$ \\
\hline Fungi & $6(0.3)$ & $5(0.8)$ & $1(0.3)$ & $1(1.0)$ & $3(2.6)$ & $7(0.3)$ & $9(1.1)^{*}$ \\
\hline \multicolumn{8}{|l|}{ Polymicrobial $^{\mathrm{d}}$} \\
\hline Including MRSA & $146(8.0)$ & $57(9.5)$ & $43(11.0)$ & $10(10.1)$ & $9(7.9)$ & $189(8.5)$ & $76(9.4)$ \\
\hline With Pseudomonas (no MRSA) & $145(7.9)$ & $52(8.7)$ & $42(10.7)$ & $13(13.1)$ & $15(13.2)$ & $187(8.4)$ & $80(9.9)$ \\
\hline No MRSA or Pseudomonas & $749(41.0)$ & $191(32.0)^{* * * *}$ & $178(45.4)$ & $41(41.4)$ & $41(36.0)$ & $927(41.8)$ & $273(33.7)^{* * * *}$ \\
\hline MRSA (mono or poly) & $285(15.6)$ & $111(18.6)$ & $69(17.6)$ & $21(21.2)$ & $22(19.3)$ & $354(15.9)$ & $154(19.0)^{*}$ \\
\hline
\end{tabular}

${ }^{\mathrm{a}} \mathrm{SSI}$, surgical site infection at any anatomic location; ${ }^{\mathrm{b}}$ monomicrobial, i.e. only one microbial isolate found on culture; ${ }^{\mathrm{c}} \mathrm{CoN}$, coagulasenegative; ${ }^{d}$ polymicrobial, i.e. two or more microbial isolates found on culture

${ }^{*} p<0.05, * * p<0.01, * * * p<0.001$ and $* * * * p<0.0001$

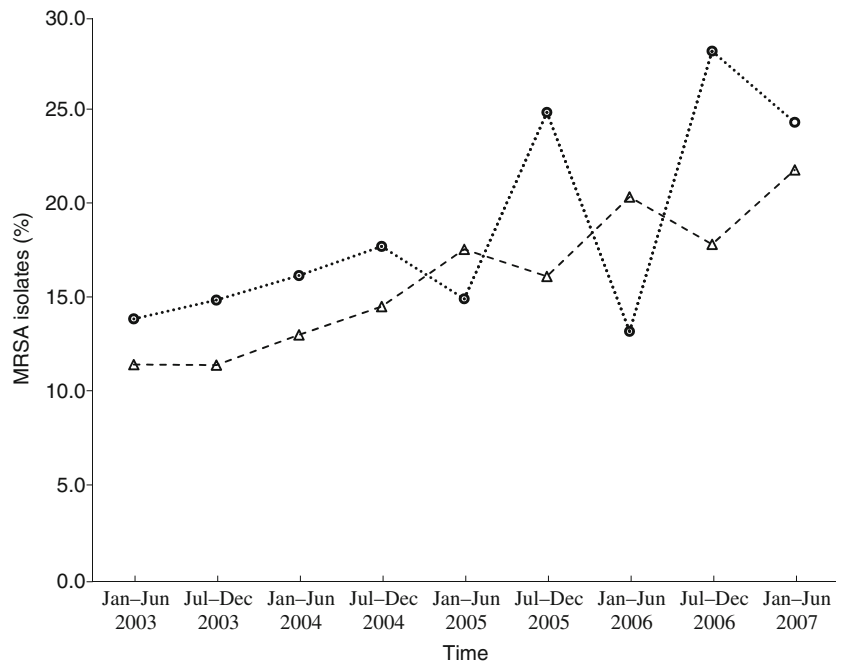

Fig. 1 Percentage of MRSA isolates in monomicrobial or polymicrobial cultures from infections involving the foot vs non-foot (or surgical) sites in hospitalised persons with diabetes during the study period. Dotted line, percentage of admissions with MRSA isolates vs total number of admissions in non-foot (or surgical) site infections group; $p<0.01$ Cochran-Armitage trending test. Dashed line, percentage with MRSA isolates as above, but for foot infections group; $p<$ 0.0001 Cochran-Armitage trending test increase being seen for both foot and non-foot infections. Cultures also yielded a variety of aerobic gram-negative isolates, albeit in a relatively small number of cases. The low rate of isolation of obligate anaerobes $(0.5 \%)$ is compatible with findings in other studies of SSTIs [43], but may also reflect the fact that in non-study situations clinicians often submit suboptimal specimens for anaerobic culture. As expected in these diabetic patients, foot wounds usually had polymicrobial cultures, but over half of nonfoot SSTIs were also polymicrobial. About $9 \%$ of all polymicrobial cultures included either MRSA or P. aeruginosa, organisms that usually require specifically targeted antibiotic therapy, rather than the usually selected empirical regimens.

We identified significant independent risk factors for mortality, including increased severity of illness on admission and having an infection other than cellulitis. A nonfoot location of diagnosed infection was associated with greater illness severity than foot infections, but was not an independent predictor of outcomes in multivariable analysis, probably because of various confounding associations. Factors significantly associated with greater hospital length of stay and costs included higher severity of illness or being 
Table 3 Relationship of variables to mortality rates, length of stay and hospital cost in univariate analysis

\begin{tabular}{|c|c|c|c|c|}
\hline Variable & $n$ & Mortality, $n(\%)$ & Length of stay in days, mean (SD) & Cost per US\$, mean (SD) \\
\hline All patients & 3,030 & $41(1.4)$ & $8.4(7.9)$ & $12,065(18,879)$ \\
\hline Live discharge & 2,989 & & $8.3(7.7)^{* * * *}$ & $11,823(18,479)^{* *}$ \\
\hline Death during hospitalisation & 41 & & $14.2(14.6)$ & $29,711(34,005)$ \\
\hline \multicolumn{5}{|l|}{ Admission illness severity } \\
\hline Severity 1 st quartile & 758 & $0(0.0)^{* * * *}$ & $5.7(4.6)^{* * * *}$ & $7,231(8,020)^{* * * *}$ \\
\hline Severity 2 nd quartile & 758 & $5(0.7)$ & $7.0(5.4)$ & $9,388(10,159)$ \\
\hline Severity 3rd quartile & 757 & $11(1.5)$ & $9.0(7.3)$ & $12,591(13,637)$ \\
\hline Severity 4 th quartile & 757 & $25(3.3)$ & $11.9(11.1)$ & $19,049(31,507)$ \\
\hline \multicolumn{5}{|l|}{ Hospitalisation and transfer status } \\
\hline Prior admission $\leq 30$ days previously & 284 & $5(1.8)$ & $8.9(7.4)^{*}$ & $12,729(12,322)^{*}$ \\
\hline Transferred from acute-care hospital & 27 & $2(7.4)$ & $12.4(11.0)^{* * *}$ & $20,922(21,988)^{* *}$ \\
\hline Transferred from skilled nursing facility & 105 & $4(3.8)$ & $11.1(8.4)^{* * *}$ & $14,663(11,559)^{* * * *}$ \\
\hline \multicolumn{5}{|l|}{ Type of infection } \\
\hline Cellulitis & 2,425 & $23(0.9)^{* * *}$ & $7.8(6.7)^{* * * *}$ & $10,946(18,118)^{* * * *}$ \\
\hline Ulcer and other infection & 491 & $11(2.2)$ & $9.6(8.7)$ & $14,696(17,080)$ \\
\hline Surgical site & 114 & $7(6.1)$ & $15.7(17.4)$ & $24,658(32,226)$ \\
\hline \multicolumn{5}{|l|}{ Anatomic location of infection } \\
\hline Foot & 2,220 & $23(1.0)^{*}$ & $8.1(6.7)$ & $11,640(18,202)$ \\
\hline Non-foot or any surgical site & 810 & $18(2.2)$ & $9.2(10.5)$ & $13,242(20,605)$ \\
\hline \multicolumn{5}{|l|}{ Monomicrobial $^{\mathrm{a}}$} \\
\hline MSSA & 427 & $4(0.9)$ & $7.2(6.9)^{* * *}$ & $11,103(32,419)^{* * * *}$ \\
\hline MRSA & 243 & $4(1.6)$ & $8.1(7.9)$ & $10,877(15,345)$ \\
\hline Coagulase-negative staphylococci & 171 & $0(0)$ & $6.9(5.4)^{* * *}$ & $8,973(8,645)^{* *}$ \\
\hline Streptococcus spp. & 195 & $1(0.5)$ & $7.9(6.9)$ & $11,919(17,627)$ \\
\hline Enterococcus spp. & 64 & $1(1.6)$ & $10.3(9.8)$ & $12,808(11,778)$ \\
\hline Other gram-positives & 12 & $0(0)$ & $6.2(3.5)$ & $8,880(6,872)$ \\
\hline Enterobacter & 13 & $0(0)$ & $6.3(4.0)$ & $9,747(6,575)$ \\
\hline P. aeruginosa & 46 & $1(2.2)$ & $8.3(6.7)$ & $12,182(15,778)$ \\
\hline Other gram-negatives & 95 & $7(7.4)^{* * *}$ & $11.1(10.1)^{* *}$ & $15,778(18,450)^{* *}$ \\
\hline Anaerobic bacteria & 16 & $0(0)$ & $8.8(7.7)$ & $10,607(9,616)$ \\
\hline Fungi & 16 & $1(6.3)$ & $6.9(4.3)$ & $9,425(8,342)$ \\
\hline \multicolumn{5}{|l|}{ Polymicrobial $^{\mathrm{b}}$} \\
\hline Including MRSA & 265 & $1(0.4)$ & $9.9(12.8)^{*}$ & $14,109(26,917)^{* *}$ \\
\hline Including Pseudomonas without MRSA & 267 & $9(3.4)^{* *}$ & $9.9(8.2)^{* * * *}$ & $14,820(14,089)^{* * * *}$ \\
\hline Without MRSA or Pseudomonas & 1,200 & $12(1.0)$ & $8.3(6.8)$ & $11,817(12,881)$ \\
\hline MRSA (mono or poly) & 508 & $5(1.0)$ & $9.0(10.8)$ & $12,564(22,191)$ \\
\hline Major surgery at index hospitalisation & 297 & $7(2.4)$ & $10.3(9.1)^{* * * *}$ & $16,834(17,821)^{* * * *}$ \\
\hline
\end{tabular}

${ }^{a}$ Monomicrobial, i.e. only one microbial isolate found on culture; ${ }^{b}$ polymicrobial, i.e. two or more microbial isolates found on culture ${ }^{*} p<0.05, * * p<0.01, * * * p<0.001$ and $* * * * p<0.0001$

transferred from another acute care hospital or skilled nursing facility. These factors have been classified previously as healthcare-associated infections and associated with adverse clinical and economic consequences [37, 38]. We also found that diagnosed infections with monomicrobial isolation of an aerobic gram-negative organism or polymicrobial cultures that included $P$. aeruginosa also increased length of stay and costs. In contrast, neither polymicrobial cultures with MRSA, nor a non-foot location of infection were independently associated with adverse outcomes when controlled for clinical severity and other confounding factors. Because MRSA isolation among these patients was much more common than $P$. aeruginosa or other gram-negatives isolation, anti-MRSA treatment might 
Table 4 Variables associated with mortality, length of stay or hospital costs in multivariable analysis

\begin{tabular}{|c|c|c|c|c|c|c|}
\hline \multirow[t]{2}{*}{ Variable $^{\mathrm{b}}$} & \multicolumn{2}{|l|}{ Mortality $^{\mathrm{a}}$} & \multicolumn{2}{|c|}{ Length of stay } & \multicolumn{2}{|l|}{ Cost } \\
\hline & OR $(95 \% \mathrm{CI})$ & $p$ value & Beta $^{c}$ & $p$ value & Beta $^{c}$ & $p$ value \\
\hline Admission illness severity ${ }^{\mathrm{d}}$ & $1.86(1.53-2.26)$ & $<0.0001$ & 0.210 & $<0.0001$ & 0.210 & $<0.0001$ \\
\hline Transferred from another acute-care hospital & $5.08(1.01-25.68)$ & 0.0492 & 0.422 & 0.0154 & 0.422 & 0.005 \\
\hline Major surgery during index hospitalisation & $1.26(0.52-3.01)$ & 0.6096 & 0.341 & 0.0002 & 0.341 & $<0.0001$ \\
\hline Ulcer compared with cellulitis & $1.21(0.56-2.64)$ & 0.6308 & 0.086 & 0.9595 & 0.086 & 0.0299 \\
\hline Surgical site infection compared with cellulitis & $3.54(1.42-8.85)$ & 0.0067 & 0.452 & $<0.0001$ & 0.452 & $<0.0001$ \\
\hline Other (i.e. non-Pseudomonas) gram-negative monomicrobial & $8.86(3.56-22.03)$ & $<0.0001$ & 0.244 & 0.0005 & 0.244 & 0.0031 \\
\hline Pseudomonas polymicrobial (no-MRSA) & $3.13(1.40-7.00)$ & 0.0054 & 0.184 & 0.0025 & 0.184 & 0.0003 \\
\hline
\end{tabular}

${ }^{\mathrm{a}} \mathrm{C}$-statistic for logistic mortality rate model was 0.84

${ }^{\mathrm{b}}$ Variables with $p$ value significant in at least one model

${ }^{\mathrm{c}}$ The coefficient generated by multivariable model using log scale of length of stay or cost as an outcome measure. The model fit $R^{2}$ was 0.16 and 0.19 , respectively

${ }^{\mathrm{d}}$ Admission illness severity $=$ the logit transformation of predicted probability of death on admission based on multivariable logistic regression models

have been more prompt and effective. Unfortunately, we were unable to further investigate this issue because our database lacked pharmacy data.

Our literature review uncovered very few previous studies that have compared SSTIs involving the foot vs non-foot sites in diabetic patients. In one prospective study of treatment of complicated skin and skin structure infections, clinical success rates were non-significantly lower in diabetic patients with an infected foot ulcer $(\sim 68 \%)$ than in those with other types of infections ( $85 \%)$ [44]. In another prospective treatment study of patients with complicated skin and skin structure infections, the proportion of patients who were clinically cured was similar for those with a diabetic foot infection $(\sim 85 \%)$ and those with other types of infection ( 90\%) [31]. Finally, another treatment trial of patients with a complicated SSTI found that the $37 \%$ of patients with diabetes were older and more likely to have impaired renal function at enrolment. Complex abscesses were less common in diabetic than in non-diabetic patients, while cellulitis and infected ulcers were more common [45].

Limitations of our study include the fact that analyses were retrospective and our observations constrained by the information available in the database. We were unable to analyse differences between various medical or surgical treatments and their associated outcomes, nor could we determine whether deaths were directly attributable to the SSTI. Moreover, some SSTI patients may have had a negative wound culture and been excluded from our study. In addition, if specimens for culture from an open wound are collected by swab, the results may represent contamination or colonisation, rather than infection. Nevertheless, clinicians rarely culture clinically uninfected wounds, and this study was conducted before culturing to detect MRSA colonisation was common. As all patients had a diagnosis of SSTI that was sufficiently severe to warrant hospitalisation, the possibility that the wounds were uninfected seems remote. Nevertheless, associations between wound isolates and outcomes are not necessarily causal. Another limitation is that we did not investigate nosocomial infections for the index hospitalisation, since we only included cultures collected within $48 \mathrm{~h}$ of admission. Hence, our findings may not necessarily be generalisable for hospital-acquired infections. Finally, hospitals included in the study were predominantly in the northeast region of the USA, which may not be representative of other regions in the USA or other countries.

Our data add important observations relating to the sparsely studied but common clinical problem of SSTI in persons with diabetes. The study cohort was large and all patients had positive culture isolates. Perhaps our most noteworthy microbiological finding is that only MRSA increased in prevalence as an isolate; this probably reflects epidemiological trends in the locales of the hospitals included. We identified significant independent risk factors for longer length of stay, higher costs and mortality rates during the admission. Our findings that patients with wounds containing MRSA tended to be more severely ill at clinical presentation may have clinical implications leading to earlier diagnosis and treatment. We would like to see our observations replicated in other populations, especially with prospectively designed studies. Meanwhile, being alert to the risk factors for adverse outcomes, which are easily identified on admission, could allow hospital staff to concentrate efforts on those diabetic patients at high risk of adverse outcomes. 
Acknowledgements This work was presented in part at the Interscience Conference on Antimicrobial Agents and Chemotherapy of the Infectious Diseases Society of America, 46th Annual Meeting, 25 October 2008, in Washington, DC, USA. We thank the following staff of CareFusion Clinical Research for their contribution to statistical analyses, and their technical and administrative support: X. Sun, K. Derby, V. Gupta and E. Falk.

Duality of interest B. A. Lipsky and J. A. Weigelt served as consultants for Ortho-McNeil Janssen Scientific Affairs, LLC, Raritan, NJ, USA. Y. P. Tabak, R. S. Johannes and L. Hyde are employees of CareFusion, Marlborough, MA, USA. L. Vo is an employee of Ortho-McNeil Janssen Scientific Affairs. This study was supported by Ortho-McNeil Janssen Scientific Affairs, LLC.

\section{References}

1. Kao LS, Knight MT, Lally KP, Mercer DW (2005) The impact of diabetes in patients with necrotizing soft tissue infections. Surg Infect (Larchmt) 6:427-438

2. Sendi P, Johansson L, Norrby-Teglund A (2008) Invasive group B Streptococcal disease in non-pregnant adults: a review with emphasis on skin and soft-tissue infections. Infection 36:100-111

3. Homer-Vanniasinkam S (2007) Surgical site and vascular infections: treatment and prophylaxis. Int J Infect Dis 11(Suppl 1):S17-S22

4. DiNubile MJ, Lipsky BA (2004) Complicated infections of skin and skin structures: when the infection is more than skin deep. J Antimicrob Chemother 53(Suppl 2):ii37-ii50

5. Lipsky BA, Berendt AR, Deery HG et al (2004) Diagnosis and treatment of diabetic foot infections. Clin Infect Dis 39:885-910

6. Rajagopalan S (2005) Serious infections in elderly patients with diabetes mellitus. Clin Infect Dis 40:990-996

7. Frykberg RG, Zgonis T, Armstrong DG et al (2006) Diabetic foot disorders. A clinical practice guideline (revision 2006). J Foot Ankle Surg 45:S1-S66

8. Bader MS (2008) Diabetic foot infection. Am Fam Physician 78:71-79

9. Pallin DJ, Egan DJ, Pelletier AJ, Espinola JA, Hooper DC, Camargo CA Jr (2008) Increased US emergency department visits for skin and soft tissue infections, and changes in antibiotic choices, during the emergence of community-associated methicillin-resistant Staphylococcus aureus. Ann Emerg Med 51:291-298

10. Bertoni AG, Saydah S, Brancati FL (2001) Diabetes and the risk of infection-related mortality in the U.S. Diabetes Care 24:10441049

11. Shah BR, Hux JE (2003) Quantifying the risk of infectious diseases for people with diabetes. Diabetes Care 26:510-513

12. U.S. Department of Health and Human Services, Centers for Disease Control and Prevention (2007) National diabetes fact sheet: general information and national estimates on diabetes in the United States, 2007. Available from www.cdc.gov/diabetes/ pubs/pdf/ndfs_2007.pdf, accessed 8 September 2009

13. Lipsky BA (2004) Medical treatment of diabetic foot infections. Clin Infect Dis 39(Suppl 2):S104-S114

14. Kirby JT, Mutnick AH, Jones RN, Biedenbach DJ, Pfaller MA (2002) Geographic variations in garenoxacin (BMS284756) activity tested against pathogens associated with skin and soft tissue infections: report from the SENTRY Antimicrobial Surveillance Program (2000). Diagn Microbiol Infect Dis 43:303-309

15. Whitman TJ (2008) Community-associated methicillin-resistant Staphylococcus aureus skin and soft tissue infections. Dis Mon 54:780-786
16. Liu C, Graber CJ, Karr M et al (2008) A population-based study of the incidence and molecular epidemiology of methicillinresistant Staphylococcus aureus disease in San Francisco, 2004 2005. Clin Infect Dis 46:1637-1646

17. Klein E, Smith DL, Laxminarayan R (2007) Hospitalizations and deaths caused by methicillin-resistant Staphylococcus aureus, United States, 1999-2005. Emerg Infect Dis 13:1840-1846

18. Anderson DJ, Sexton DJ, Kanafani ZA, Auten G, Kaye KS (2007) Severe surgical site infection in community hospitals: epidemiology, key procedures, and the changing prevalence of methicillinresistant Staphylococcus aureus. Infect Control Hosp Epidemiol 28:1047-1053

19. Cantlon CA, Stemper ME, Schwan WR, Hoffman MA, Qutaishat SS (2006) Significant pathogens isolated from surgical site infections at a community hospital in the Midwest. Am J Infect Control 34:526-529

20. Crum NF, Lee RU, Thornton SA et al (2006) Fifteen-year study of the changing epidemiology of methicillin-resistant Staphylococcus aureus. Am J Med 119:943-951

21. Fridkin SK, Hageman JC, Morrison M et al (2005) Methicillinresistant Staphylococcus aureus disease in three communities. N Engl J Med 352:1436-1444

22. Moran GJ, Amii RN, Abrahamian FM, Talan DA (2005) Methicillin-resistant Staphylococcus aureus in communityacquired skin infections. Emerg Infect Dis 11:928-930

23. Nather A, Bee CS, Huak CY et al (2008) Epidemiology of diabetic foot problems and predictive factors for limb loss. J Diabetes Complications 22:77-82

24. Vardakas KZ, Horianopoulou M, Falagas ME (2008) Factors associated with treatment failure in patients with diabetic foot infections: an analysis of data from randomized controlled trials. Diabetes Res Clin Pract 80:344-351

25. Stanaway S, Johnson D, Moulik P, Gill G (2007) Methicillinresistant Staphylococcus aureus (MRSA) isolation from diabetic foot ulcers correlates with nasal MRSA carriage. Diabetes Res Clin Pract 75:47-50

26. Tentolouris N, Petrikkos G, Vallianou N et al (2006) Prevalence of methicillin-resistant Staphylococcus aureus in infected and uninfected diabetic foot ulcers. Clin Microbiol Infect 12:186-189

27. Game F, Jeffcoate W (2004) MRSA and osteomyelitis of the foot in diabetes. Diabet Med 21(Suppl 4):16-19

28. Dang CN, Prasad YD, Boulton AJ, Jude EB (2003) Methicillinresistant Staphylococcus aureus in the diabetic foot clinic: a worsening problem. Diabet Med 20:159-161

29. Wagner A, Reike H, Angelkort B (2001) Highly resistant pathogens in patients with diabetic foot syndrome with special reference to methicillin-resistant Staphylococcus aureus infections. Dtsch Med Wochenschr 126:1353-1356

30. Dryden MS (2009) Skin and soft tissue infection: microbiology and epidemiology. Int J Antimicrob Agents 34(Suppl 1):S2-S7

31. Noel GJ, Bush K, Bagchi P, Ianus J, Strauss RS (2008) A randomized, double-blind trial comparing ceftobiprole medocaril with vancomycin plus ceftazidime for the treatment of patients with complicated skin and skin-structure infections. Clin Infect Dis 46:647-655

32. Weigelt J, Itani K, Stevens D, Lau W, Dryden M, Knirsch C (2005) Linezolid vs vancomycin in treatment of complicated skin and soft tissue infections. Antimicrob Agents Chemother 49: $2260-2266$

33. Graham DR, Lucasti C, Malafaia O et al (2002) Ertapenem once daily vs piperacillin-tazobactam 4 times per day for treatment of complicated skin and skin-structure infections in adults: results of a prospective, randomized, double-blind multicenter study. Clin Infect Dis 34:1460-1468

34. McKinnon PS, Sorensen SV, Liu LZ, Itani KM (2006) Impact of linezolid on economic outcomes and determinants of cost in a 
clinical trial evaluating patients with MRSA complicated skin and soft-tissue infections. Ann Pharmacother 40:1017-1023

35. Iezzoni LI, Moskowitz MA (1988) A clinical assessment of MedisGroups. JAMA 260:3159-3163

36. Lipsky BA, Weigelt JA, Gupta V, Killian A, Peng MM (2007) Skin, soft tissue, bone, and joint infections in hospitalized patients: epidemiology and microbiological, clinical, and economic outcomes. Infect Control Hosp Epidemiol 28: $1290-1298$

37. Kollef MH, Shorr A, Tabak YP, Gupta V, Liu LZ, Johannes RS (2005) Epidemiology and outcomes of health-care-associated pneumonia: results from a large US database of culture-positive pneumonia. Chest 128:3854-3862

38. Shorr AF, Tabak YP, Killian AD, Gupta V, Liu LZ, Kollef MH (2006) Healthcare-associated bloodstream infection: a distinct entity? Insights from a large U.S. database. Crit Care Med 34:2588-2595

39. Tabak YP, Johannes RS, Silber JH (2007) Using automated clinical data for risk adjustment: development and validation of six disease-specific mortality predictive models for pay-forperformance. Med Care 45:789-805
40. U.S. Department of Health and Human Services, Centers for Medicare \& Medicaid Services (2008). Available from www.cms. hhs.gov, accessed 17 April 2008

41. Duan N (1983) Smearing estimate: a nonparametric retransformation method. J Am Stat Assoc 78:605-610

42. Efron B, Tibshirani R (1993) An introduction to the bootstrap. Chapman \& Hall, London

43. Rennie RP, Jones RN, Mutnick AH (2003) Occurrence and antimicrobial susceptibility patterns of pathogens isolated from skin and soft tissue infections: report from the SENTRY Antimicrobial Surveillance Program (United States and Canada, 2000). Diagn Microbiol Infect Dis 45:287-293

44. Lipsky BA, Stoutenburgh U (2005) Daptomycin for treating infected diabetic foot ulcers: evidence from a randomized, controlled trial comparing daptomycin with vancomycin or semisynthetic penicillins for complicated skin and skin-structure infections. J Antimicrob Chemother 55:240-245

45. Embil JM, Soto NE, Melnick DA (2006) A post hoc subgroup analysis of meropenem vs imipenem/cilastatin in a multicenter, double-blind, randomized study of complicated skin and skin-structure infections in patients with diabetes mellitus. Clin Ther 28:1164-1174 